\title{
Experimental investigation of the transition between Autler-Townes splitting and electromagnetically-induced-transparency models
}

\author{
L. Giner, ${ }^{1}$ L. Veissier, ${ }^{1}$ B. Sparkes, ${ }^{2}$ A. S. Sheremet, ${ }^{1,3}$ A. Nicolas, ${ }^{1}$ O. S. Mishina, ${ }^{4}$ M. Scherman, ${ }^{1,5}$ S. Burks, ${ }^{1}$ \\ I. Shomroni, ${ }^{6}$ D. V. Kupriyanov, ${ }^{3}$ P. K. Lam, ${ }^{2}$ E. Giacobino, ${ }^{1}$ and J. Laurat ${ }^{1,}$ \\ ${ }^{1}$ Laboratoire Kastler Brossel, Université Pierre et Marie Curie, Ecole Normale Supérieure, CNRS, 4 place Jussieu, \\ 75252 Paris Cedex 05, France \\ ${ }^{2}$ Centre for Quantum Computation and Communication Technology, Australian National University, Canberra, ACT 0200, Australia \\ ${ }^{3}$ Department of Theoretical Physics, State Polytechnic University, 195251, St. Petersburg, Russia \\ ${ }^{4}$ Universität des Saarlandes, Theoretische Physik, 66123 Saarbrücken Germany \\ ${ }^{5}$ ONERA, The French Aerospace Lab, Chemin de la Hunière, 91761 Palaiseau, France \\ ${ }^{6}$ Chemical Physics Department, Faculty of Chemistry, Weizmann Institute of Science, Rehovot, Israel
}

(Received 25 June 2012; published 22 January 2013)

\begin{abstract}
Two phenomena can affect the transmission of a probe field through an absorbing medium in the presence of an additional field: electromagnetically induced transparency (EIT) and Autler-Townes splitting (ATS). Being able to discriminate between the two is important for various practical applications. Here we present an experimental investigation into a method that allows for such a disambiguation as proposed by Anisimov, Dowling, and Sanders in Phys. Rev. Lett. 107, 163604 (2011). We apply the proposed test based on Akaike's information criterion to a coherently driven ensemble of cold cesium atoms and find a good agreement with theoretical predictions, therefore demonstrating the suitability of the method. Beyond the applicability of the test, our results demonstrate that the transition features are highly sensitive to the properties of the medium under study, potentially providing a practical characterizing tool for complex systems.
\end{abstract}

DOI: 10.1103/PhysRevA.87.013823

PACS number(s): 42.50.Gy, 03.67.-a, 42.50.Ct

Fine engineering of interactions between light and matter is critical for various purposes, including information processing and high-precision metrology. For more than two decades, coherent effects leading to quantum interference in the amplitudes of optical transitions have been widely studied in atomic media, opening the way to controlled modifications of their optical properties [1]. More specifically, such processes as coherent population trapping [2,3] or electromagnetically induced transparency (EIT) [4-6] allow one to take advantage of the modification of an atomic system by a so-called control field to change the transmission characteristics of a probe field. These features are especially important for the implementation of optical quantum memories [7] relying on dynamic EIT [8], or for coherent driving of a great variety of systems, ranging from superconducting circuits [9] to nanoscale optomechanics [10].

However, if in general the transparency of an initially absorbing medium for a probe field is increased by the presence of a control field, two very different processes can be invoked to explain it in a $\Lambda$-type configuration. One of them is a quantum Fano interference between two paths in a three-level system [11], which occurs even at very low control intensity and gives rise to EIT [12]. The other one is the appearance of two dressed states in the excited level at large control intensity, corresponding to the Autler-Townes splitting (ATS) [13-15]. Discerning whether a transparency feature observed in an absorption profile is the signature of EIT or ATS is crucial and a long-standing issue [16-19].

In this endeavor, a recent theoretical study by Anisimov, Dowling and Sanders [20] introduced a method to discriminate between these two phenomena. In this paper, we report on an

\footnotetext{
*julien.laurat@upmc.fr
}

experimental implementation of the proposed criterion. Based on a detailed analysis of the absorption profile of a probe field in an atomic ensemble of cold cesium atoms in the presence of a control field, our study demonstrates in a quantitative way the transition between the ATS and the EIT models when the control field power is decreased. The observed features of the transition turned out to be very sensitive to the specific medium properties. As an example of such a dependence, we will show how the multilevel structure of the atomic system and some residual inhomogeneous broadening translate into the observed transition.

The experimental setup is illustrated in Fig. 1(a). The optically thick atomic ensemble is obtained from cold cesium atoms in a magneto-optical trap (MOT). The three-level $\Lambda$ system involves the two ground states, $|g\rangle=\left|6 S_{1 / 2}, F=3\right\rangle$ and $|s\rangle=\left|6 S_{1 / 2}, F=4\right\rangle$, and one excited state $|e\rangle=\mid 6 P_{3 / 2}, F=$ $4\rangle$. The control field is resonant with the $|s\rangle$ to $|e\rangle$ transition, while the probe field is scanned around the $|g\rangle$ to $|e\rangle$ transition, with a detuning $\delta$ from resonance.

Each run of the experiment involves a period for the cold atomic cloud to build up and a period for measurement. This sequence is repeated every $25 \mathrm{~ms}$ and controlled with a fieldprogrammable gate array (FPGA) board. After the build-up of the cloud in the MOT, the current in the coils generating the trapping magnetic field and then the MOT trapping beams are switched off. In order to transfer the atoms from the $|s\rangle$ to the $|g\rangle$ ground state, the MOT is illuminated with a $\sigma^{-}$polarized 1-ms-long depump pulse with a power of $900 \mu \mathrm{W}$ and resonant with the $|s\rangle$ to $|e\rangle$ transition. After this preparation stage, the optical depth at resonance for atoms in $|s\rangle$ is zero within our experimental precision. The remaining spurious magnetic fields have been canceled down to $5 \mathrm{mG}$ using a RF spectroscopy technique. 
(a)

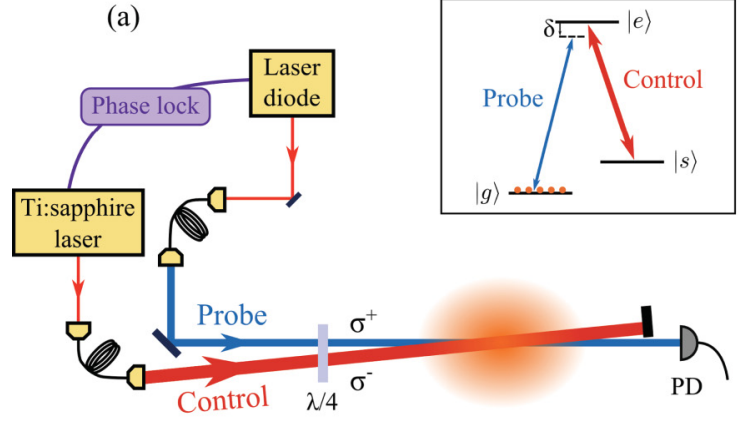

(b)
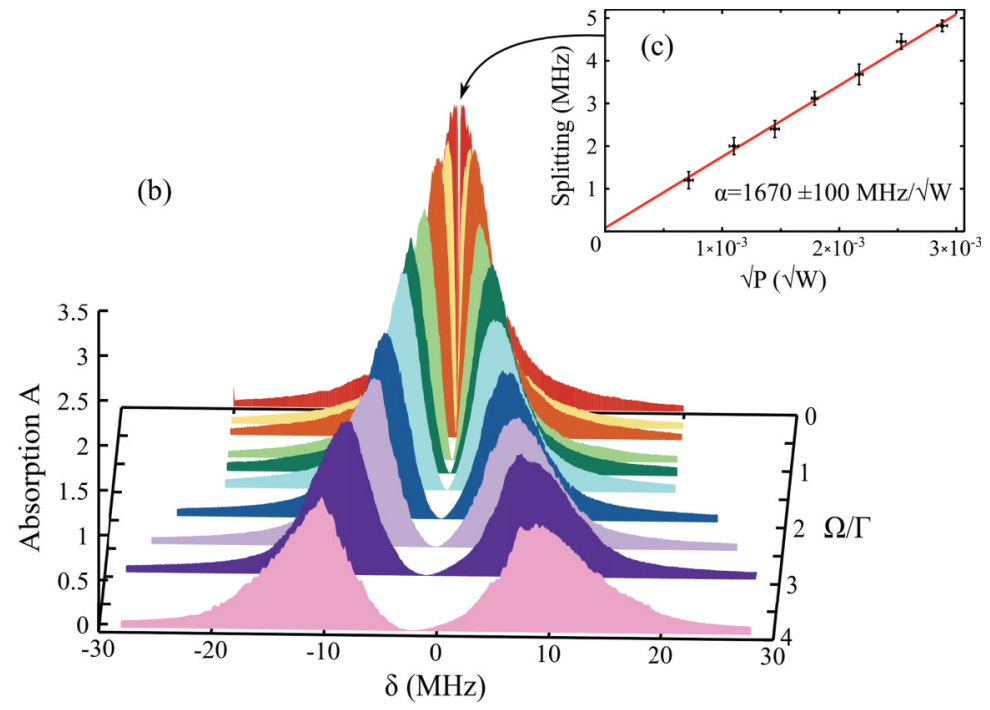

FIG. 1. (Color online) Absorption in a $\Lambda$-type system. (a) Experimental setup: a weak probe beam and a control beam travel through a cloud of cold cesium atoms. The atoms are initially in the ground state $|g\rangle$. The probe field is close to the $|g\rangle \rightarrow|e\rangle$ transition (detuning $\delta$ ) while the control field drives the $|s\rangle \rightarrow|e\rangle$ transition on resonance. PD is a high-gain photodiode. (b) Absorption profiles are displayed as a function of the detuning $\delta$ for a control Rabi frequency $\Omega$ between $0.1 \Gamma$ and $4 \Gamma$, where $\Gamma$ is the natural linewidth. (c) Experimental splitting as a function of $\sqrt{P}$, where $P$ is the measured control power, fit by a linear function.

The measurement period starts $3 \mathrm{~ms}$ after the extinction of the MOT magnetic field. The atomic ensemble is illuminated with a $30-\mu$ s-long control pulse and a probe pulse lasting $15 \mu \mathrm{s}$ is sent during this time. The probe field is emitted by an extended cavity grating stabilized laser diode, whereas the control field is generated by a Ti:sapphire laser locked on resonance using saturated absorption spectroscopy. The two lasers are phase locked. The control field is $\sigma^{-}$polarized, with a $200 \mu \mathrm{m}$ waist in the MOT and a $2^{\circ}$ angle relative to the direction of the probe beam. The probe field is $\sigma^{+}$polarized, with a waist of $50 \mu \mathrm{m}$ and a power of $30 \mathrm{nW}$. To measure absorption profiles, the probe beam frequency is swept over a few natural linewidths by changing the locking frequency point. Its absorption is measured with a high-gain photodiode. The optical depth in the $|g\rangle$ state is chosen to be around 3 to avoid any profile shape distortion due to the limited dynamic range of the photodiode.

Figure 1(b) gives the absorption of the probe field, $A=$ $\ln \left(I_{\text {ref }} / I\right)$, as a function of its detuning $\delta$ from resonance for different values of the control power $(0.1$ to $200 \mu \mathrm{W})$, i.e., for different values of the control Rabi frequency $\Omega$. The quantity $I_{\text {ref }}$, which gives the transmission in the absence of atoms, is measured by sending an additional probe pulse when all the atoms are still in the $|s\rangle$ ground state. The Rabi frequency $\Omega$ of the control field is changed from very weak values (at the back) to four times the natural linewidth $\Gamma$ (at the front). Each profile results from an averaging over twenty repetitions of the experiment. The narrow transparency dip appearing for low Rabi frequencies gets wider when the Rabi frequency increases, to finally give two well-separated resonances corresponding to the two excited dressed states.

Let us note that the Rabi frequency $\Omega$ is a linear function of the electric field and can be expressed as $\Omega=\alpha \sqrt{P}$, with $P$ being the power of the control field. An effective value of $\Omega$ can be inferred from the experimental splittings (i.e., the distance between the two maxima) observed in the absorption profiles for low-power control field [Fig. 1(c)]. For a three-level system this splitting is indeed equal to the Rabi frequency to within a very good approximation for low decoherence in the ground state [3]. We find $\alpha=1670 \pm 100 \mathrm{MHz} / \sqrt{\mathrm{W}}$.

We now turn to the detailed analysis of the absorption profiles. For a three-level $\Lambda$ system, to first order in the probe electric field, the atomic susceptibility on the probe transition for a control field on resonance is given by $[1,17]$

$$
\chi(\delta)=-\frac{n_{g}\left|d_{e g}\right|^{2}}{\hbar \epsilon_{0}} \frac{\delta+i \gamma_{\mathrm{gs}}}{\delta^{2}-\left|\Omega_{0}\right|^{2} / 4-\gamma_{e g} \gamma_{\mathrm{gs}}+i \delta\left(\gamma_{e g}+\gamma_{\mathrm{gs}}\right)},
$$

where $n_{g}$ stands for the atomic density in state $|g\rangle$ and $d_{e g}$ denotes the electric dipole moment between $|e\rangle$ and $|g\rangle$. Here, the Rabi frequency of the control field is $\Omega_{0}=2\left|d_{e s}\right| \varepsilon_{c} / \hbar$, with $\varepsilon_{c}$ being the amplitude of the positive-frequency part of the control field. The optical coherence relaxation rate is $\gamma_{e g}=$ $\Gamma / 2$ where $\Gamma / 2 \pi=5.2 \mathrm{MHz} . \gamma_{\mathrm{gs}}$ is the dephasing rate of the ground state coherence; $\gamma_{\mathrm{gs}}=10^{-2} \Gamma$ in our experimental case.

Depending on the value of the control Rabi frequency $\Omega_{0}$, Eq. (1) can be rewritten in different ways [17-19]. For Rabi frequencies $\Omega_{0}<\Omega_{t}=\gamma_{e g}-\gamma_{\mathrm{gs}}$, the spectral poles of the susceptibility are imaginary. Then, the linear absorption $A \propto \operatorname{Im}[\chi]$ can be expressed as the difference between two Lorentzian profiles centered at zero frequency: a broad one and a narrow one. For $\Omega_{0}>\Omega_{t}$, this decomposition is not possible anymore. For large Rabi frequencies, $\Omega_{0} \gg \Gamma$, Eq. (1) can be written as the sum of two well-separated Lorentzian profiles with similar widths. Absorption profiles for these two models can thus be written as

$$
A_{\mathrm{EIT}}=\frac{C_{+}}{1+(\delta-\epsilon)^{2} /\left(\gamma_{+}^{2} / 4\right)}-\frac{C_{-}}{1+\delta^{2} /\left(\gamma_{-}^{2} / 4\right)},
$$




$$
A_{\mathrm{ATS}}=\frac{C_{1}}{1+\left(\delta+\delta_{1}\right)^{2} /\left(\gamma_{1}^{2} / 4\right)}+\frac{C_{2}}{1+\left(\delta-\delta_{2}\right)^{2} /\left(\gamma_{2}^{2} / 4\right)},
$$

where $C_{+}, C_{-}, C_{1}, C_{2}$ are the amplitudes of the Lorentzian curves, $\gamma_{+}, \gamma_{-}, \gamma_{1}$, and $\gamma_{2}$ are their respective widths, and $\epsilon, \delta_{1}$, and $\delta_{2}$ are the shifts from zero frequency. Equation (2) describes a Fano interference and corresponds to the EIT model, while Eq. (3) corresponds a strongly driven regime with a splitting of the excited state, i.e., ATS.

For a three-level system, the various parameters introduced in the two above expressions can be calculated from Eq. (1). Conversely, in our experimental system, we use functions $A_{\text {EIT }}$ and $A_{\text {ATs }}$ to fit the experimental absorption curves, adjusting all the aforementioned parameters. The test proposed in Ref. [20] aims at determining which of these generic models is the most likely for given experimental data.

Figure 2 shows the measured probe absorption as a function of the detuning $\delta$ (blue dots) together with the fits to $A_{\text {EIT }}$ (red curves) and $A_{\mathrm{ATS}}$ (green curves). A low value of the control Rabi frequency, $\Omega=0.2 \Gamma$ is shown in Fig. 2(a), and a larger one, $\Omega=2.3 \Gamma$ in Fig. 2(b). Let us note that for the EIT model a detuning parameter $\epsilon$ was introduced between the atomic line center and the EIT dip to account for a possible experimental inaccuracy in the frequency locking reference of the lasers. For the ATS model the parameters describing each Lorentzian curve are independent of each other [contrary to what would be deduced from Eq. (1)] in order to account for their experimentally different widths and heights. These asymmetries are discussed below. As expected, the EIT model fits better the low-power control field region [Fig. 2(a)] while the ATS model fits better the strong-power control field region [Fig. 2(b)].

As proposed in Ref. [20], in order to quantitatively test the quality of these model fits, we then calculate the Akaike information criterion (AIC) [21]. This criterion, directly provided
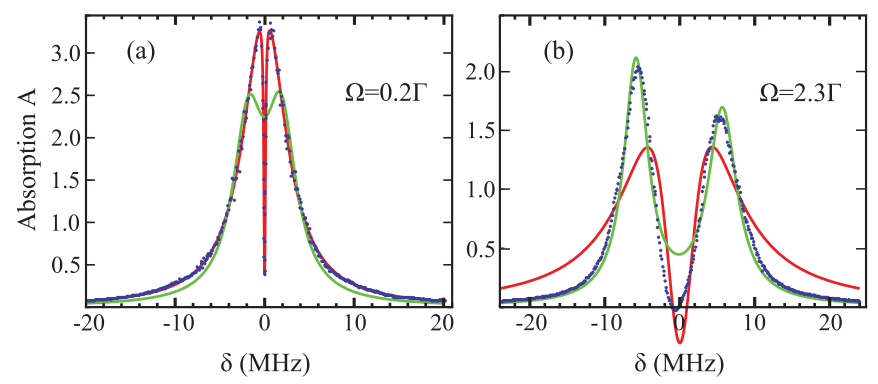

FIG. 2. (Color online) Absorption profiles and model fits for two values of the control Rabi frequency $\Omega$. Experimental data (blue dots) are presented together with the best fits of functions $A_{\mathrm{EIT}}\left(C_{+}, C_{-}, \epsilon, \gamma_{+}, \gamma_{-}\right)$(red solid lines) and $A_{\mathrm{ATS}}\left(C_{1}, C_{2}, \delta_{1}, \delta_{2}, \gamma_{1}, \gamma_{2}\right)$ (green solid lines). Parameters $C_{+}, C_{-}, C_{1}, C_{2}$ representing the amplitudes of the absorption curves are in dimensionless units, while the parameters $\epsilon, \gamma_{+}, \gamma_{-}$ and $\delta_{1}, \delta_{2}, \gamma_{1}, \gamma_{2}$ representing detunings and widths are in MHz. (a) $\Omega=0.2 \Gamma$. In this case $A_{\text {EIT }}\left(3.52,3.14,1.45 \times 10^{-2}\right.$, $5.71,0.239)$ fits the experimental data much better than $A_{\text {ATS }}(2.01,2.04,1.84,1.84,4.14,4.08) . \quad($ b) $\Omega=2.3 \Gamma$. Here $A_{\text {ATS }}(2.05,1.64,5.86,5.67,3.94,4.68)$ fits the data better than $A_{\text {EIT }}\left(1.59 \times 10^{5}, 1.59 \times 10^{5}, 1.45 \times 10^{-7}, 8.17,8.17\right)$.

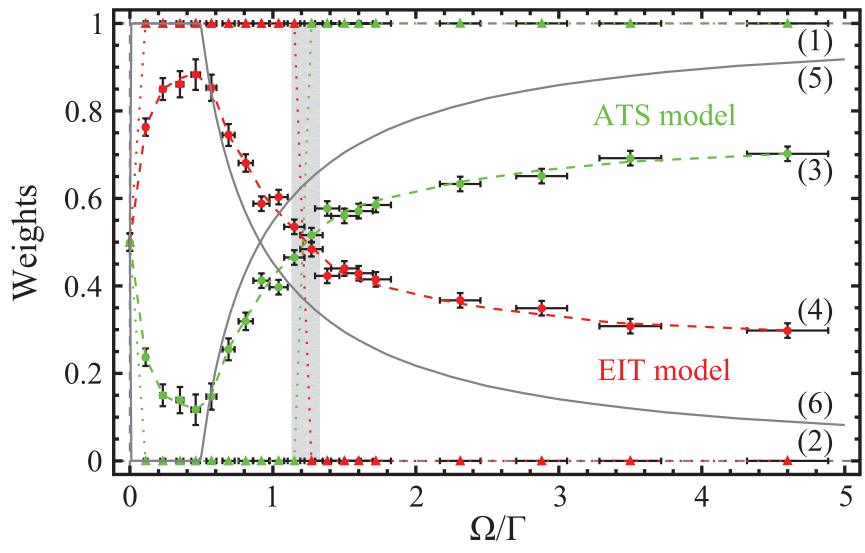

FIG. 3. (Color online) Experimental Akaike weights $w_{j}$ as a function of Rabi frequency $\Omega$ for ATS model [green triangles, curve (1)] and for EIT model [red triangles, curve (2)]. Experimental per-point weights $\bar{w}_{j}$ for ATS model [green dots, curve (3)] and for EIT model [red dots, curve (4)]. The grey area indicates the EIT-ATS model transition. Error bars include the uncertainty on the coefficient $\alpha$ and on the measured power. The solid lines give the theoretical per-point weights for a pure three-level system [curves (5) and (6)].

by the function NonLinearModelFit in MATHEMATICA, is equal to $I_{j}=2 k-\ln \left(L_{j}\right)$ where $k$ is the number of parameters used and $L_{j}$ is the maximum of the likelihood function obtained from the considered model, labeled with $j$ ( $j=$ EIT or ATS). The relative weights $w_{\text {EIT }}$ and $w_{\text {ATS }}$ that give the relative probabilities of finding one of the two models can be calculated from these quantities and are given by

$$
w_{\mathrm{EIT}}=\frac{e^{-I_{\mathrm{ETT}} / 2}}{e^{-I_{\mathrm{ETT}} / 2}+e^{-I_{\mathrm{ATS}} / 2}}, \quad w_{\mathrm{ATS}}=1-w_{\mathrm{EIT}} .
$$

These weights are plotted in Fig. 3 [curves (1) and (2)], as a function of the experimentally determined Rabi frequency. They exhibit a binary behavior. They are close to 0 or 1 and there is an abrupt transition from the EIT model to the ATS model.

We then investigate the second criterion proposed in Ref. [20], also based on Akaike's information criterion but with a mean per-point weight $\bar{w}$. It can be obtained by dividing $I_{j}$ by the number $N$ of experimental points. The weights for the EIT and the ATS models are now given respectively by

$$
\bar{w}_{\mathrm{EIT}}=\frac{e^{-I_{\mathrm{EIT}} / 2 N}}{e^{-I_{\mathrm{EIT}} / 2 N}+e^{-I_{\mathrm{ATS}} / 2 N}}, \quad \bar{w}_{\mathrm{ATS}}=1-\bar{w}_{\mathrm{EIT}} .
$$

The resulting curves are presented in Fig. 3 [curves (3) and (4)]. Starting from a per-point weight equal to 0.5 for both models in the absence of control field (the two models are equally likely), the EIT model first dominates in the low Rabi frequency region. Then the likelihood of the EIT model decreases and a crossing is observed for the same value as for the previous criterion. The ATS model then dominates for larger Rabi frequency, as expected. For the Akaike weights, as well as for the per-point weights, the behavior is in good qualitative agreement with the predictions given in Ref. [20] and with our simulations for a three-level system. 
However, a closeup analysis reveals significant deviations. The transition between the two models is obtained experimentally for $\Omega / \Gamma=1.23 \pm 0.10$, while a value of $\Omega / \Gamma=0.91$ is obtained for the per-point weights of a pure three-level system, calculated for the same Rabi frequencies, as shown in Fig. 3 (solid grey lines). Moreover, for large Rabi frequencies the per-point weights corresponding to ATS and EIT saturate at 0.7 and 0.3 , respectively, instead of going to 1 and 0 as in the theoretical three-level model. For low Rabi frequencies, the shape of the curves also differs significantly. These various features result from the fact that the system cannot be described by a simple three-level model. Below, we proceed to theoretical simulations including additional parameters that influence the ATS-EIT model transition and the general shape of the per-point weight curves.

First, we take into account the other hyperfine sublevels of the $6 P_{3 / 2}$ manifold, based on a previous theoretical model $[22,23]$. We find that these contributions explain the asymmetry between the two dressed-state resonances observed in Fig. 2(b) at large Rabi frequencies, but they do not significantly influence the per-point weight curves. The latter are shown in Fig. 4(b) (solid lines), with a crossing point for $\Omega / \Gamma=0.91$.

We then consider the effect of the Zeeman structure. Several Zeeman sublevels are involved in each atomic level, as shown in Fig. 4(a). We have determined the atomic distribution in the Zeeman sublevels from the optical pumping due to the

(a)

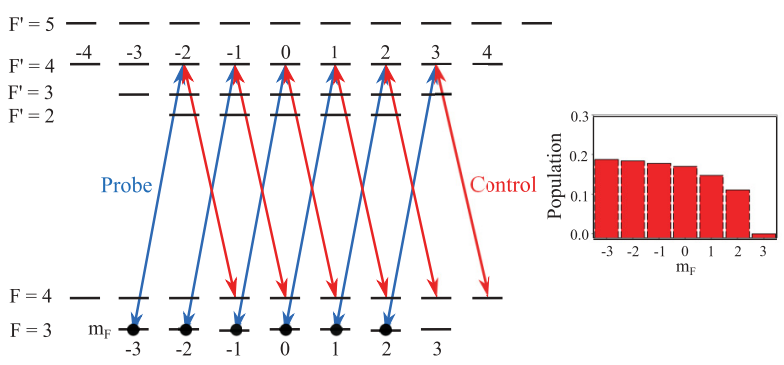

(b)

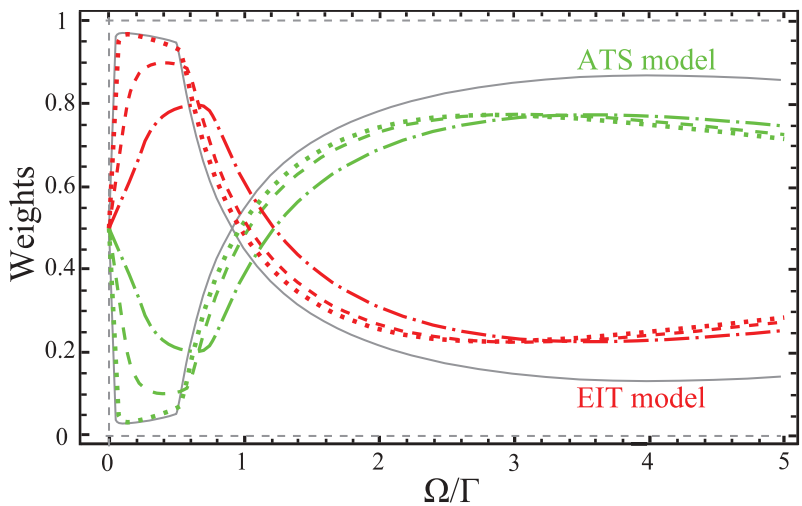

FIG. 4. (Color online) Theoretical simulations with Zeeman sublevels and Doppler broadening. (a) Level scheme for the Cs $D_{2}$ line and the six three-level transitions involving Zeeman sublevels. Inset shows the population distribution. (b) Per-point weights for a system with two ground states and taking into account all the hyperfine sublevels of the $6 P_{3 / 2}$ manifold (gray solid lines), for a system involving additionally Zeeman sublevels (dotted lines), for a system involving Zeeman sublevels and residual Doppler broadenings $\Gamma_{\mathrm{D}} /(2 \pi)=0.6 \mathrm{MHz}$ (dashed lines) and $\Gamma_{\mathrm{D}} /(2 \pi)=1.3 \mathrm{MHz}$ (dash-dotted lines). depump field [Fig. 4(a), inset]. Since the control and probe fields have opposite circular polarizations, we can consider that the atomic scheme is a superposition of six independent $\Lambda$ subsystems with different Rabi frequencies. The susceptibility is calculated as the sum of the corresponding susceptibilities. The per-point weights for theoretical absorption curves calculated from this model (including the hyperfine structure) are shown in Fig. 4(b) (dotted lines). For the horizontal axis, as the system does not have a single Rabi frequency; we have used an effective Rabi frequency obtained from the splitting between the maxima of the theoretical absorption curves. The transition point is found for $\Omega / \Gamma=0.98$, close to the value obtained for a three-level system. These simulations show that taking into account the Zeeman sublevels does not lead to a large enough alteration of the crossing point as compared with the three-level model. However, a significant change in the values of the per-point weights for large Rabi frequencies is obtained for the model including the Zeeman sublevels, and it is comparable to the experimental one.

We finally include a residual inhomogeneous Doppler broadening $\Gamma_{\mathrm{D}}$. By fitting the experimental absorption profile in the absence of control field, we obtain $\Gamma_{\mathrm{D}} /(2 \pi)=0.6 \mathrm{MHz}$. The per-point weights for theoretical absorption curves including this residual broadening are given in Fig. 4(b) (dashed lines). The crossing point is found for a value $\Omega / \Gamma=1.05$, which is in better agreement with the experimental value. The slightly larger value of the experimental transition point is very likely to be due to heating and additional broadening caused by the control laser. If we assume an inhomogeneous broadening $\Gamma_{\mathrm{D}} /(2 \pi)=1.3 \mathrm{MHz}$ (dash-dotted lines), the per-point weights for the theoretical absorption curves cross each other for $\Omega / \Gamma=1.23$. Moreover, the shape of the curves including even a small Doppler broadening agrees much better with the experimental results for the low control Rabi frequency region. Thus, including in the model both the Zeeman structure of the atomic system and a residual Doppler broadening due to the finite temperature of the atoms allows us to explain the observed experimental behavior when the EIT-ATS discrimination criterion is applied.

In summary, we have tested and analyzed in detail the transition from the ATS model to the EIT model in a well-controlled experimental situation. The criteria have been calculated and give a consistent conclusion for discerning between the two regions. We have also interpreted the observed differences from the three-level model by a refined model taking into account the specific level structure and some residual inhomogeneous broadening. In addition to its discriminating capability, this study reveals the sensitivity of the proposed test to the specific properties of the medium.

This work was supported by the ERA-NET CHIST-ERA (QScale), by the ERA-Net.RUS (Nanoquint), by the Australian Research Council Centre of Excellence for Quantum Computation and Communication Technology (CE110001027), and by the CNRS-RFBR collaboration (CNRS 6054 and RFBR P2-02-91056). A.S. acknowledges the support from the Foundation "Dynasty" and O.S.M. acknowledges support from the Ile-de-France program IFRAF. J.L. is a member of the Institut Universitaire de France. 
[1] M. Fleischhauer, A. Imamoğlu, and J. P. Marangos, Rev. Mod. Phys. 77, 633 (2005).

[2] G. Alzetta et al., Nuovo Cimento B 36, 5 (1976).

[3] E. Arimondo, Coherent Population Trapping in Laser Spectroscopy, Prog. Opt. (Elsevier, Amsterdam, 1996), Vol. 5, pp. 257-354.

[4] S. E. Harris, J. E. Field, and A. Imamoğlu, Phys. Rev. Lett. 64, 1107 (1990).

[5] K.-J. Boller, A. Imamoğlu, and S. E. Harris, Phys. Rev. Lett. 66, 2593 (1991).

[6] J. P. Marangos, J. Mod. Opt. 45, 471 (1998).

[7] A. I. Lvovsky, B. C. Sanders, and W. Tittel, Nat. Photonics 3, 706 (2009).

[8] L. V. Hau, S. E. Harris, Z. Dutton, and C. H. Behroozi, Nature (London) 397, 594 (1999).

[9] W. R. Kelly, Z. Dutton, J. Schlafer, B. Mookerji, T. A. Ohki, J. S. Kline, and D. P. Pappas, Phys. Rev. Lett. 104, 163601 (2010).

[10] A. H. Safavi-Naeini et al., Nature (London) 472, 69 (2011).

[11] U. Fano, Phys. Rev. 124, 1866 (1961).

[12] S. Harris, Phys. Today 50, 36 (1997).
[13] S. H. Autler and C. H. Townes, Phys. Rev. 100, 703 (1955).

[14] C. Cohen-Tannoudji and S. Reynaud, J. Phys. B 10, 2311 (1977).

[15] C. Cohen-Tannoudji, J. Dupont-Roc, and G. Grynberg, AtomPhoton Interactions: Basic Processes and Applications (Wiley Interscience, New York, 1992).

[16] T. Abi Salloum et al., J. Mod. Opt. 54, 2459 (2007).

[17] P. Anisimov and O. Kocharovskaya, J. Mod. Opt. 55, 3159 (2008).

[18] T. Y. Abi-Salloum, Phys. Rev. A 81, 053836 (2010).

[19] Z. H. Li, Y. Li, Y. F. Dou, and J. X. Zhang, Chin. Phys. B 21, 034204 (2012)

[20] P. M. Anisimov, J. P. Dowling, and B. C. Sanders, Phys. Rev. Lett. 107, 163604 (2011).

[21] K. P. Burnham and D. R. Anderson, Model Selection and Multimodel Inference, 2nd ed. (Springer-Verlag, New York, 2002).

[22] O. S. Mishina, M. Scherman, P. Lombardi, J. Ortalo, D. Felinto, A. S. Sheremet, A. Bramati, D. V. Kupriyanov, J. Laurat, and E. Giacobino, Phys. Rev. A 83, 053809 (2011).

[23] M. Scherman et al., Opt. Express 20, 4346 (2012). 